\title{
Impact of oversedation prevention in ventilated critically ill patients: a randomized trial-the AWARE study
}

SRLF Trial Group*

\begin{abstract}
Background: Although oversedation has been associated with increased morbidity in ventilated critically ill patients, it is unclear whether prevention of oversedation improves mortality. We aimed to assess 90 -day mortality in patients receiving a bundle of interventions to prevent oversedation as compared to usual care.

Methods: In this randomized multicentre trial, all adult patients requiring mechanical ventilation for more than $48 \mathrm{~h}$ were included. Two groups were compared: patients managed according to usual sedation practices (control), and patients receiving sedation according to an algorithm which provided a gradual multilevel response to pain, agitation, and ventilator dyssynchrony with no specific target to alter consciousness and no use of sedation scale and promoted the use of alternatives to continuous infusion of midazolam or propofol (intervention).

Results: Inclusions were stopped before reaching the planned enrolment. Between 2012 and 2014, 584 patients were included in the intervention group and 590 in the control group. Baseline characteristics were well balanced between groups. Although the use of midazolam and propofol was significantly lower in the intervention group, 90 -day mortality was not significantly lower (39.4 vs. $44.2 \%$ in the control group, $p=0.09$ ). There were no significant differences in 1-year mortality between the two groups. The time to first spontaneous breathing trial and time to successful extubation were significantly shorter in the intervention group than in the control group. These last results should be interpreted with precaution regarding the several limitations of the trial including the early termination.
\end{abstract}

Conclusions: This underpowered study of severely ill patients was unable to show that a strategy to prevent oversedation could significantly reduce mortality.

Trial registration NCT01617265

Keywords: Intensive care units, Mechanical ventilation, Sedation, Mortality, Weaning

\section{Background}

Intravenous hypnotics, often combined with morphinics, are commonly used in mechanically ventilated patients in the intensive care unit (ICU) to control pain, agitation, and ensure synchrony with the ventilator [1]. However, the continuous infusion of midazolam or propofol often results in oversedation [2]. Factors involved in oversedation are multiple, including drug pharmacokinetic and

*Correspondence: Bdejonghe@chi-poissy-st-germain.fr

Société de Réanimation de Langue Française (SRLF), 48 Avenue Claude Vellefaux, 75010 Paris, France

Full list of author information is available at the end of the article pharmacodynamic properties, inadequate objectives in terms of consciousness, and lack of frequent reassessment of patient condition and hypnotic needs. Oversedation has been associated with prolonged mechanical ventilation and higher rates of nosocomial infections, ICU-acquired weakness, and delirium.

Strategies developed to avoid oversedation have been based either on (1) the use of continuous intravenous hypnotics combined with daily interruption of sedatives every $24 \mathrm{~h} \mathrm{[3],} \mathrm{(2)} \mathrm{the} \mathrm{continuous} \mathrm{titration} \mathrm{of} \mathrm{hypnotics}$ according to predefined goals of comfort and consciousness, with frequent patient assessments and prescription changes [4-7], or (3) the first-intention use of alternatives 
to continuous intravenous midazolam or propofol [810]. These strategies were associated with a reduction in mechanical ventilation duration. Few observational studies have assessed the impact of an oversedation prevention strategy on mortality [10-13]. In a prospective observational study, Shehabi et al. reported that patients with deep sedation (indicated by at least one measurement of Richmond Agitation Sedation Scale [RASS] -3 to -5 ) during the first $48 \mathrm{~h}$ of mechanical ventilation were more likely to die in the ICU than patients with a lighter sedation (RASS -2 to +1 ), independently of age, comorbidities, and severity of acute illness [11]. To the best of our knowledge, this observation has not been confirmed in a randomized trial.

The main objective of the present randomized controlled trial was to determine whether a strategy aiming to prevent oversedation could reduce 90-day mortality in critically ill patients requiring mechanical ventilation compared to usual care.

\section{Methods}

In accordance with French law, the study was approved by the institutional review board of Clermont-Ferrand, France, and by the Ethics Committee of the French Intensive Care Society (SRLF, Société de Réanimation de Langue Française). Informed consent or deferred consent was obtained from each patient or his/her legal surrogate. An independent data safety monitoring board had full access to the unblinded data.

\section{Participants and settings}

The study conducted by the SRLF Trial Group was planned as a parallel two-group individually randomized trial. Patients were eligible if they were aged 18 years or more, were receiving invasive mechanical ventilation for $<12 \mathrm{~h}$, and had an expected invasive mechanical ventilation duration $>48 \mathrm{~h}$ after randomization. Patients admitted after cardiac arrest, those with neuromuscular disease, tracheostomy, severe intracranial hypertension, status epilepticus, decision to withdraw care, or considered moribund were not included.

\section{Oversedation prevention (OSP)}

The OSP strategy was centred on the identification of patients' level of agitation, ventilator asynchrony, and pain, on a 4-level scale, with gradual on-demand responses, frequent reassessments, and promotion of alternatives to continuous around-the-clock intravenous hypnotics infusion (Fig. 1). The alternatives to continuous infusion of midazolam or propofol in the intervention group were other benzodiazepines, antipsychotic agents, zolpidem, and hydroxyzine.
There was no aim to alter consciousness, and therefore, no sedation scale was used. However, consciousness alteration could result from treatment of existing agitation, existing ventilator dyssynchrony, or existing or anticipated pain. During two national training and education meetings, the OSP strategy was explained to investigators (usually an ICU doctor and nurse), who in turn implemented the OSP strategy in their own centre. OSP strategy posters were used at the bedside.

\section{Usual care}

Patients in the control group were treated according to the routine sedation practices used in each participating centre, reported in an pre-study survey of sedation practices in France [14]. In both intervention and control groups, the use of dexmedetomidine was not permitted. In both groups, pain was measured according to the current practice in each participating centre. Weaning was conducted according to the French ICU Society guidelines [15].

\section{Outcomes}

The primary study endpoint was 90-day mortality after randomization. Secondary endpoints were day-28, hospital and 1-year mortality, time from randomization to first spontaneous breathing trial, time to successful extubation (defined as absence of invasive mechanical ventilation for 48 consecutive hours). Other outcome criteria are reported in Additional file 1: Appendix 1.

\section{Randomization, allocation concealment, and blinding}

Using a secure, computer-generated, interactive, webresponse system available at each study centre, patients were randomly assigned in a 1:1 ratio to study groups. Randomization was stratified by centre using permutation blocks of variable sizes. Sequences were generated by a biostatistician not involved in patient recruitment. Investigators had no access to the randomization list and were not aware of the size of the randomization blocks. Given the very nature of the assessed intervention, blinding of the physicians and nurses was not feasible [16-18]. However, the primary outcome (death at day 90) is an objective one, which counterbalances this lack of blinding [19].

\section{Sample size}

We assumed a mortality rate of $22 \%$ in the control group at day 90 . To show a $5 \%$ absolute reduction in 90-day mortality in the OSP group, with a two-sided type I error of $5 \%$ and a power of $90 \%$, the planned enrolment was 2720 patients. 


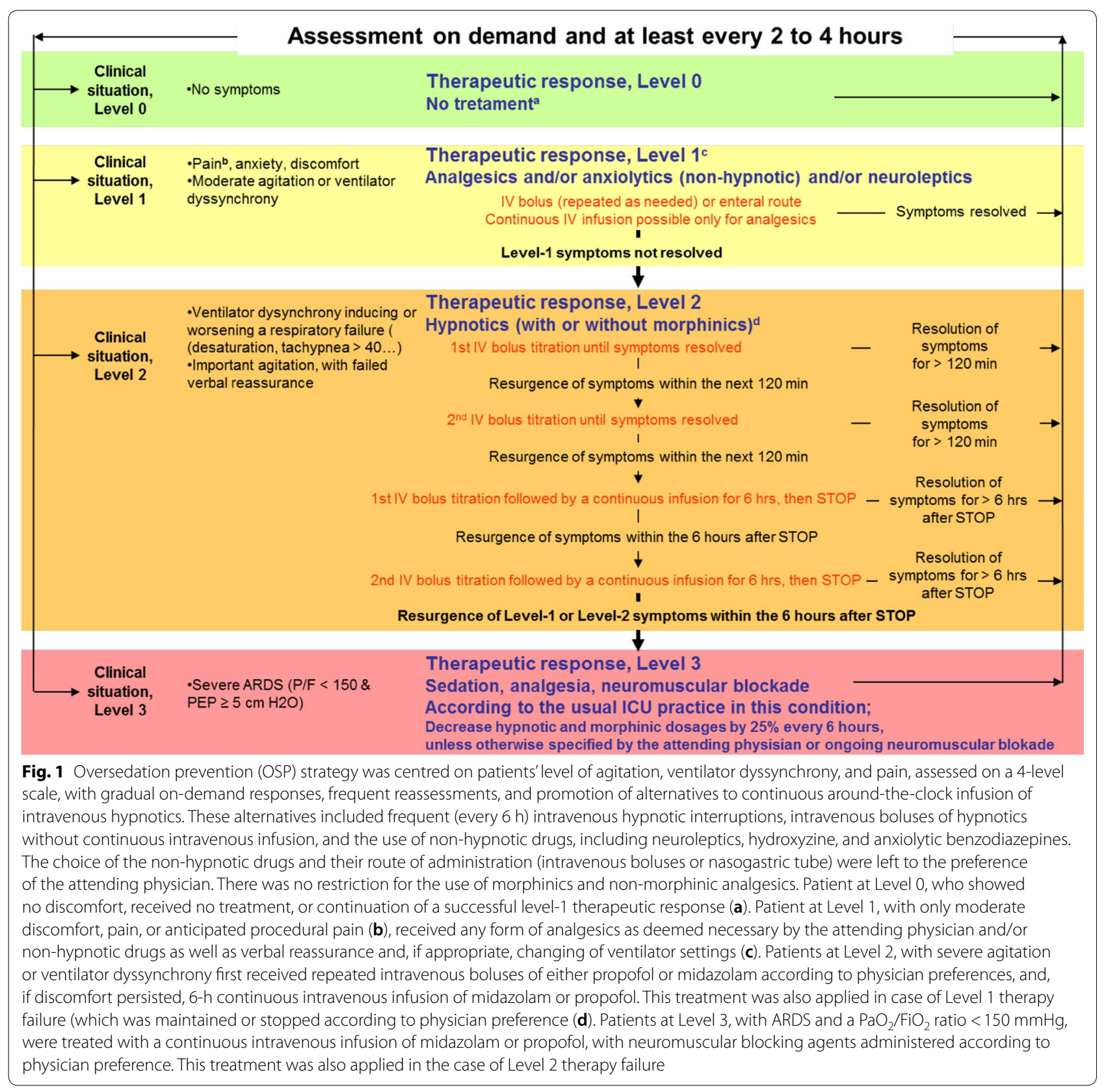

\section{Statistical analysis}

Statistical analyses were conducted in the intentionto-treat population. Data were described using counts and percentages or means and standard deviations (or median and interquartile range). Death proportions were compared using the Chi-square test, and mechanical ventilation-free days were compared using the Wilcoxon test. Time-dependent events were analysed using competing risk models taking into account death and extubation. Description of the occurrence of these events was made using cumulative incidence curves. Cumulative incidence curves were compared between the two groups with the Fine and Gray test; hazard ratios (HRs) and their $95 \%$ confidence intervals (95\% CIs) were estimated by the competing risk models [20]. For the two patients (one in the OSP group and one in the control group) lost to follow-up for the primary outcome (90-day mortality), imputation of missing data (alive status) was performed. A two-tailed $p$ value $<0.05$ was considered statistically significant. Statistical analyses were performed using SAS version 9.4 (SAS Institute Inc) and R version 3.2.2 [21]. 


\section{Results}

\section{Baseline characteristics}

Forty-six ICUs were involved in the study. Of those, 18 (39.1\%) were university-affiliated, 15 (32.6\%) were medical ICUs, and 31 (67.4\%) medical surgical ICUs. Between July 2012 and July 2014, 1179 patients were included and randomized. The nurse/patient ratio was 2.5. The trial was stopped because of a decreasing average per centre recruitment rate, despite considerable help and encouragement. Five patients withdrew their consent and were therefore excluded from analysis, as requested by French law. Two patients (one in each group) were lost for the 90-day follow-up and were arbitrarily considered alive (Fig. 2), leaving 584 patients in the OSP group and 590 in the control group available for the main analysis.

Table 1 shows the patients' baseline characteristics, which were similar between the two groups. In the OSP and control groups, mean age (standard deviation) was 66 (13) and 67 (14), mean simplified acute physiology score II (SAPS II) was 53.6 (17.8) and 54.4 (18.6), and 54.5 and $53.0 \%$ of the patients were receiving norepinephrine at randomization, respectively. Of note, chronic psychotropic medication use did not differ between the two groups, as shown in Table 1 . At randomization, more than $65 \%$ of the patients were comatose (deep sedation or unarousable), as shown in Table 1. Sedation levels at randomization are reported in Additional file 1: Appendix 2.

\section{Outcomes}

At day-90, 230 patients (39.4\%) had died in the OSP group and $261(44.2 \%)$ in the control group, $p=0.09$. Of note those mortality rates were far higher than the initial sample size calculation of the trial. There were also no significant differences in day-28 (Table 2), in-hospital (Fig. 3), and 1-year mortality between the two groups (Table 2). Cumulative dosages of intravenous propofol and midazolam were significantly lower in the OPS group (Table 2). Cumulative dosage of intravenous sufentanil was significantly lower in the OPS group, whereas there

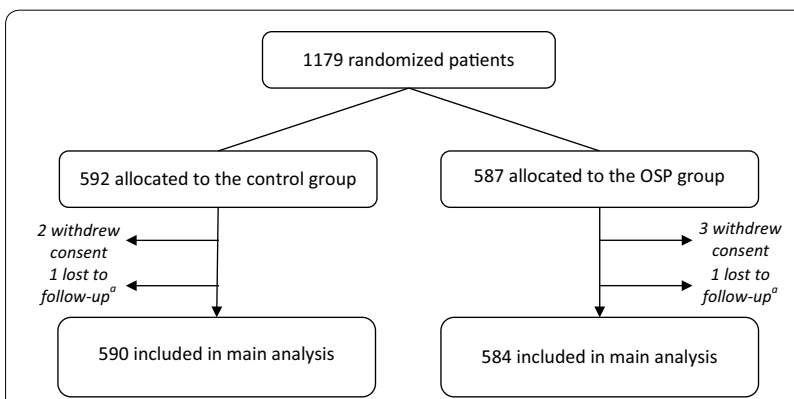

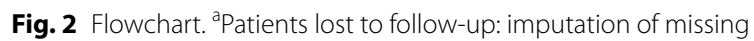
data (alive vital status) was performed. OSP, oversedation prevention was no significant difference in cumulative dosages of other morphinics between the two groups (Table 2).

First spontaneous breathing trial occurred significantly earlier in the OSP group than in the control group (HR 1.18 [1.03-1.36], $p=0.015$ (Additional file 1: Appendix 3, Figure). Similarly, successful extubation occurred significantly earlier in the OSP group than in the control group (HR 1.15 [1.02-1.31], $p=0.03$ (Additional file 1: Appen$\operatorname{dix} 3$, Figure).

There was no significant difference in the other secondary outcomes, i.e. time to first sitting in a chair, time to first standing by the bed, presence of proximal muscle weakness, delirium, length of stay in the ICU (Table 2). Self-extubation was significantly more frequent in the OSP group than in the control group (70 vs. 48 events, HR $1.50[1.04 ; 2.16], p=0.03)$. Percentage of patients awake on a daily assessment between day 1 and day 7 are presented in the Additional file 1: Appendix 4.

\section{Discussion}

In this multicentre randomized study, we were unable to show that a gradual multilevel bundle strategy to prevent oversedation could significantly reduce mortality of severely ill ICU patients requiring mechanical ventilation. There were no significant differences between the two groups in in-hospital and 1-year mortality. However, oversedation prevention resulted in significantly less use of intravenous midazolam and propofol, and significantly earlier weaning initiation and extubation. Last, the numerous limitations including early termination of the trial weaken the result interpretation.

We chose an OSP strategy centred on the identification of patients' level of agitation, ventilator asynchrony, and pain, on a 4-level scale, with gradual on-demand responses, frequent reassessments, and promotion of alternatives to continuous around-the-clock intravenous hypnotics (midazolam or propofol) infusion. Interestingly, in the OSP algorithm, interventions were titrated only on patients' needs to control pain, agitation, and ventilator asynchrony (except in the level 3), with no attempt to alter consciousness, even slightly, as a specific goal. Accordingly, the OSP strategy did not include the use of any sedation scale. Cumulative dosages of propofol and midazolam were significantly lower in the OPS group. We did not use dexmedetomidine as an alternative to continuous intravenous hypnotics because at the time of study design, the very recent commercialization of dexmedetomidine in France precluded homogeneous and optimal use among the participating centres [22-25].

Our study was unable to show that the OSP strategy reduced mortality compared to standard care in critically ill patients. Furthermore, mortality was high in the study population. More than $40 \%$ of the patients had died at 
Table 1 Demographic and baseline characteristics at randomization

\begin{tabular}{|c|c|c|}
\hline & Control $(n=590)$ & $\begin{array}{l}\text { Oversedation } \\
\text { prevention } \\
(n=584)\end{array}$ \\
\hline Age (years), mean (SD) & $67(14)$ & $66(13)$ \\
\hline Female gender, $n(\%)$ & $200(33.9)$ & $202(34.6)$ \\
\hline BMI (kilograms divided by height in metres squared), mean (SD) & $26.7(6.6)$ & $27.4(7.1)$ \\
\hline Chronic alcohol use, $n(\%)$ & $115(19.5)$ & $126(21.6)$ \\
\hline Chronic psychotropic medication use, $n(\%)$ & $177(30.0)$ & $166(28.4)$ \\
\hline Benzodiazepine and related medications & $110(18.6)$ & $109(18.7)$ \\
\hline Neuroleptics & $22(3.7)$ & $33(5.7)$ \\
\hline Antidepressants & $72(12.2)$ & $70(12.0)$ \\
\hline Opioid medication & $42(7.1)$ & $35(6.0)$ \\
\hline Tobacco use, $n(\%)$ & $156(26.5)$ & $169(28.9)$ \\
\hline Liver cirrhosis with ascites or oesophageal varices, $n(\%)$ & $34(5.8)$ & $37(6.3)$ \\
\hline Chronic renal replacement therapy, $n$ (\%) & $9(1.5)$ & $10(1.7)$ \\
\hline Chronic respiratory insufficiency with home oxygen therapy, $n$ (\%) & $42(7.1)$ & $51(8.7)$ \\
\hline NYHA class IV chronic heart failure, $n(\%)$ & $16(2.7)$ & $23(3.9)$ \\
\hline Barthel score before admission, median (Q1-Q3) & $100(100-100)$ & $100(95-100)$ \\
\hline \multicolumn{3}{|l|}{ Knauss chronic health status before admission, $n(\%)$} \\
\hline Normal health status & $155(26.3)$ & $152(26.0)$ \\
\hline Moderate activity limitation & $285(48.3)$ & $267(45.7)$ \\
\hline Severe activity limitation due to chronic disease & $139(23.6)$ & $157(26.9)$ \\
\hline Bedridden patient & $11(1.9)$ & $8(1.4)$ \\
\hline \multicolumn{3}{|l|}{ MacCabe class before admission, $n(\%)$} \\
\hline No fatal disease & $363(61.5)$ & $348(59.6)$ \\
\hline Ultimately fatal disease & $181(30.7)$ & $197(33.7)$ \\
\hline Rapidly fatal disease & $46(7.8)$ & $39(6.7)$ \\
\hline At home without assistance before current hospital admission, $n(\%)$ & $373(63.2)$ & $367(62.8)$ \\
\hline ICU admission SAPS II score (first 24 h), mean (SD) & $54.4(18.6)$ & $53.6(17.8)$ \\
\hline ICU admission SOFA score (first 24 h), median (Q1-Q3) & $9(7-12)$ & $9(7-12)$ \\
\hline Medical admission, $n(\%)$ & $520(88.1)$ & $530(90.8)$ \\
\hline Norepinephrine at randomization, $n(\%)$ & $312(53.0)$ & $318(54.5)$ \\
\hline Midazolam at randomization $\left(n_{1}=493, n_{2}=496\right), n(\%)$ & $412(83.6)$ & $421(84.9)$ \\
\hline Propofol at randomization $\left(n_{1}=493, n_{2}=496\right), n(\%)$ & $74(15.0)$ & $69(13.9)$ \\
\hline Severe sepsis, $n(\%)$ & $50(8.5)$ & $57(9.8)$ \\
\hline Septic shock, $n(\%)$ & $339(57.5)$ & $323(55.3)$ \\
\hline ARDS, $n(\%)$ & $186(31.5)$ & $187(32.1)$ \\
\hline \multicolumn{3}{|l|}{ ICU primary diagnosis } \\
\hline Pulmonary infection, $n(\%)$ & $246(41.7)$ & $239(40.9)$ \\
\hline Abdominal infection, $n(\%)$ & $49(8.3)$ & $54(9.3)$ \\
\hline Other Infection, $n(\%)$ & $41(6.9)$ & $57(9.8)$ \\
\hline Cardiac failure or cardiogenic pulmonary oedema, $n(\%)$ & $56(9.5)$ & $53(9.1)$ \\
\hline COPD exacerbation or acute asthma, $n(\%)$ & $51(8.6)$ & $51(8.7)$ \\
\hline Acute pancreatitis, $n$ (\%) & $9(1.5)$ & $13(2.2)$ \\
\hline Drug intoxication, $n(\%)$ & $12(2.0)$ & $9(1.5)$ \\
\hline Metabolic disorder, $n(\%)$ & $18(3.1)$ & $7(1.2)$ \\
\hline Trauma, $n(\%)$ & $7(1.2)$ & $7(1.2)$ \\
\hline Acute stroke, $n(\%)$ & $5(0.8)$ & $2(0.3)$ \\
\hline Miscellaneous, $n$ (\%) & $96(16.3)$ & $92(15.8)$ \\
\hline \multicolumn{3}{|l|}{ Sedation level on the RASS scale at randomization ${ }^{a}$} \\
\hline Very agitated, $n(\%)$ & $1(0.4)$ & $3(1.1)$ \\
\hline
\end{tabular}


Table 1 (continued)

\begin{tabular}{lcc}
\hline & Control $(\boldsymbol{n = 5 9 0 )}$ & $\begin{array}{c}\text { Oversedation } \\
\text { prevention } \\
(\boldsymbol{n}=\mathbf{5 8 4})\end{array}$ \\
\hline Agitated, $n(\%)$ & & $1(0.4)$ \\
Restless, $n(\%)$ & $4(1.5)$ & $5(1.9)$ \\
Alert and calm, $n(\%)$ & $3(1.1)$ & $12(4.6)$ \\
Drowsy, $n$ (\%) & $12(4.5)$ & $12(4.6)$ \\
Light sedation, $n(\%)$ & $12(4.5)$ & $15(5.7)$ \\
Moderate sedation, $n(\%)$ & $21(7.9)$ & $23(8.8)$ \\
Deep sedation, $n(\%)$ & $30(11.3)$ & $66(25.2)$ \\
Unarousable, $n(\%)$ & $63(23.8)$ & $125(47.7)$ \\
\hline
\end{tabular}

SD, standard deviation; BMI, body mass index; NYHA, New York Heart Association; Q1-Q3, 1st and 3rd quartiles; SAPS, Simplified Acute Physiology Score, SOFA, Sequential Organ Failure Assessment; ICU, intensive care unit, COPD, chronic obstructive pulmonary disease; ARDS, acute respiratory distress syndrome

a Sedation was measured using the RASS scale in 268 patients in the control group and 267 patients in the oversedation prevention group. The exact level on the RASS scale was not available for 3 patients in the control group and 5 patients in the oversedation prevention group.

3 months and almost $60 \%$ at 1 year. These mortality rates were much higher than anticipated at study design and higher than those reported in previous studies on light sedation strategies [1-13]. This high mortality very likely reflects the severity of the acute conditions at ICU admission, as suggested by a SAPS II score higher than commonly reported in trials on sedation $[5,8,10,25,26]$. Old age and the low rate of post-operative admissions both could have contributed to the high SAPS II. Similarly, the percentage of patients on vasoactive drugs at randomization was high.

The severity of the acute conditions of our study population compared to previous studies suggests the inclusion of patients in real-life conditions. Demonstration of the positive impact of an intervention, such as a strategy to prevent oversedation, might be difficult in patients with particularly severe admission conditions and requires a larger sample of patients. In our study, less than half of the planned included patients were finally enrolled which undoubtedly makes the trial underpowered. Furthermore, the planned inclusion number was based on a mortality rate of $22 \%$ in the control group. There is such a high difference between the a priori postulated mortality rate in the control group, and the a posteriori observed one (which is much closer to $50 \%$ ) that even in case we would have been able to recruit the planned 2720 patients, the real power of the trial would have been much lower than the $90 \%$ nominal power.

Despite the severity of the conditions of the study patients, and the above limitations, the OSP strategy resulted in significantly shorter mechanical ventilation duration. Similar findings have been observed in previous randomized studies on light sedation in less severely ill ICU populations. This finding is important, as physicians may be reluctant to adopt a light sedation strategy among the most severely ill patients. Indeed, as agitation and device removal may be perceived as particularly dangerous in this population, physicians may favour continuous intravenous sedation. The present trial did not show that oversedation prevention was associated with lower mortality, but it showed that it was associated with secondary benefits of faster weaning and extubation.

One explanation for shorter mechanical ventilation duration is provided in our study by a significantly shorter time to first spontaneous breathing trial. An adequate consciousness level is among the prerequisite criteria for physicians to initiate the weaning process leading to extubation, along with other criteria including absence of high-grade fever, low oxygen, positive expiratory endpressure, and vasoactive drug needs [27]. A light sedation strategy might promote preservation of consciousness or early return to consciousness when other weaning criteria are met [28].

Study limitations The numerous limitations including the early termination and associated lack of power weaken the results.

We did not design a weaning protocol in the control group; patients were treated according to usual practice in the participating ICUs. Unfortunately, we do not have any data showing that the French ICU weaning guidelines were applied similarly in both groups. Physicians in the participating study centres might have unconsciously changed their practice over the study period, with a progressive implementation of some aspects of the OSP strategy in control patients, further reducing the difference in sedation practices between the two groups. Insufficient compliance with the relatively novel multilevel gradual intervention might also have reduced the difference in sedation practices between the two groups. A cluster randomization at the ICU level would have 
Table 2 Outcomes

\begin{tabular}{|c|c|c|c|c|}
\hline & Control $(n=590)$ & $\begin{array}{l}\text { Oversedation } \\
\text { prevention } \\
(n=584)\end{array}$ & $P$ value & $\begin{array}{l}\text { Hazard ratio } \\
\text { ( } 95 \% \text { confidence } \\
\text { interval) }\end{array}$ \\
\hline 28-day mortality & $198(33.6)$ & $177(30.4)$ & $0.24^{\mathrm{a}}$ & \\
\hline 90-day mortality & $261(44.2)$ & $230(39.4)$ & $0.09^{\mathrm{a}}$ & \\
\hline 1-year mortality & $296(60.0)$ & $267(56.5)$ & $0.26^{\mathrm{a}}$ & \\
\hline Mechanical ventilation-free days at day 28 (days), median (Q1-Q3) & $14(0-24)$ & $16(0-24)$ & $0.36^{b}$ & \\
\hline Ventilator-associated pneumonia, $n$ & 92 & 94 & $0.79^{c}$ & $1.04(0.78 ; 1.38)$ \\
\hline Mechanical ventilation $\geq 48$ h, $n(\%)$ & $425(72.0)$ & $418(71.6)$ & $0.86^{\mathrm{a}}$ & \\
\hline Non-invasive ventilation after extubation, $n$ (\%) & $152(25.8)$ & $177(30.3)$ & $0.08^{\mathrm{a}}$ & \\
\hline $\begin{array}{l}\text { Duration of non-invasive ventilation after extubation (days), median (Q1- } \\
\text { Q3) }\end{array}$ & $2(1-4)$ & $3(2-4)$ & $0.05^{b}$ & \\
\hline Tracheostomy, $n$ & 26 & 24 & $0.81^{c}$ & $0.93(0.54 ; 1.62)$ \\
\hline Delirium, $n$ & 232 & 230 & $0.99^{c}$ & $1.00(0.84 ; 1.19)$ \\
\hline Proximal weakness after awakening, $n$ & 193 & 208 & $0.26^{c}$ & $1.11(0.92 ; 1.35)$ \\
\hline Patients with intravenous midazolam, $n(\%)$ & $464(78.6)$ & $419(71.8)$ & $0.01^{\mathrm{a}}$ & \\
\hline Cumulative dosage of midazolam (mg), median (Q1-Q3) & $263(120-660)$ & $218(72-696)$ & $0.03^{b}$ & \\
\hline Patients with intravenous propofol, $n(\%)$ & $232(39.3)$ & $214(36.6)$ & $0.34^{\mathrm{a}}$ & \\
\hline Cumulative dosage of propofol (mg), median (Q1-Q3) & $2785(645-7140)$ & $1443(120-4800)$ & $<0.001^{b}$ & \\
\hline Patients with intravenous morphinics, $n(\%)$ & $501(84.9)$ & $482(82.5)$ & $0.31^{\mathrm{a}}$ & \\
\hline Patients with IV sufentanil, $n(\%)$ & $263(44.6)$ & $241(41.3)$ & $0.28^{\mathrm{a}}$ & \\
\hline Cumulative dosage of sufentanil $(\mu \mathrm{g})$, median $(Q 1-Q 3)$ & $930(472-2592)$ & $870(280-2160)$ & $0.04^{b}$ & \\
\hline Patients with IV fentanyl, $n$ (\%) & $204(34.6)$ & $206(35.3)$ & $0.8^{\mathrm{a}}$ & \\
\hline Cumulative dosage of fentanyl $(\mu \mathrm{g})$, median $(Q 1-Q 3)$ & $4985(2400-15,445)$ & $4656(1340-16,200)$ & $0.29^{b}$ & \\
\hline Patients with IV morphine, $n(\%)$ & $73(12.4)$ & $91(15.6)$ & $0.1^{\mathrm{a}}$ & \\
\hline Cumulative dosage of morphine (mg), median (Q1-Q3) & $17.5(7-55)$ & $20(6-43)$ & $0.69^{b}$ & \\
\hline Patients with IV remifentanil, $n$ (\%) & $49(8.3)$ & $45(7.7)$ & $0.7^{a}$ & \\
\hline Cumulative dosage of remifentanil $(\mu \mathrm{g})$, median $(Q 1-Q 3)$ & $14,400(6000-28,800)$ & $7200(3000-19,200)$ & $0.05^{b}$ & \\
\hline Self-extubation, $n$ & 48 & 70 & $0.03^{c}$ & $1.50(1.04 ; 2.16)$ \\
\hline Ventricular tachycardia or fibrillation, $n$ & 18 & 21 & $0.61^{c}$ & $1.18(0.63 ; 2.11)$ \\
\hline Acute coronary syndrome or myocardial infarction, $n$ & 7 & 8 & $0.77^{c}$ & $1.16(0.42 ; 3.18)$ \\
\hline Cardiac arrest, $n$ & 20 & 13 & $0.22^{c}$ & $0.65(0.33 ; 1.31)$ \\
\hline
\end{tabular}

For comparison of time dependent events analyzed using competing risks models to take into account competing risks as death or extubation (e.g. ventilator associated pneumonia), no percentages are provided

For comparison of variables in post-randomization sub-group (e.g. ICU length of stay in survivors), no $P$ values are provided

$\mathrm{Cl}$, confidence interval; Q1-Q3, 1st and 3rd quartiles; MV, mechanical ventilation; VAP, ventilator-associated pneumonia; NIV, non-invasive ventilation; ICU, intensive care unit; LOS, length of stay

a Variables compared using the chi-square test

b Variables compared using the Wilcoxon test

c Variables analyzed using competing risks models to take into account competing risks (as death, extubation, ICU discharge, ...). For each of these outcomes, Gray test $P$ value and hazard ratio (95\% confidence interval) from competing risks models were presented

limited those group contamination issues; however, the risk of a selection bias associated with such a design was deemed greater and led us to choose an individual randomization scheme [29-32]. This point remains a strong limitation to interpret the secondary endpoints in this non-blinded study.

The gap between estimated 90-day mortality used for the sample size calculation and the higher mortality rates observed also represents an important limitation study further reducing the study power.
Another limitation is that we did not use a sedation scale to measure the effect of the OSP strategy on consciousness. This option was deliberately selected to avoid that clinicians would try to titrate IV sedatives to reach the common target of slightly altered consciousness in the intervention group, in which no specific alteration of consciousness should be targeted. Unfortunately, the surrogate markers for consciousness level used in the study (amounts of sedatives used, single daily assessment 


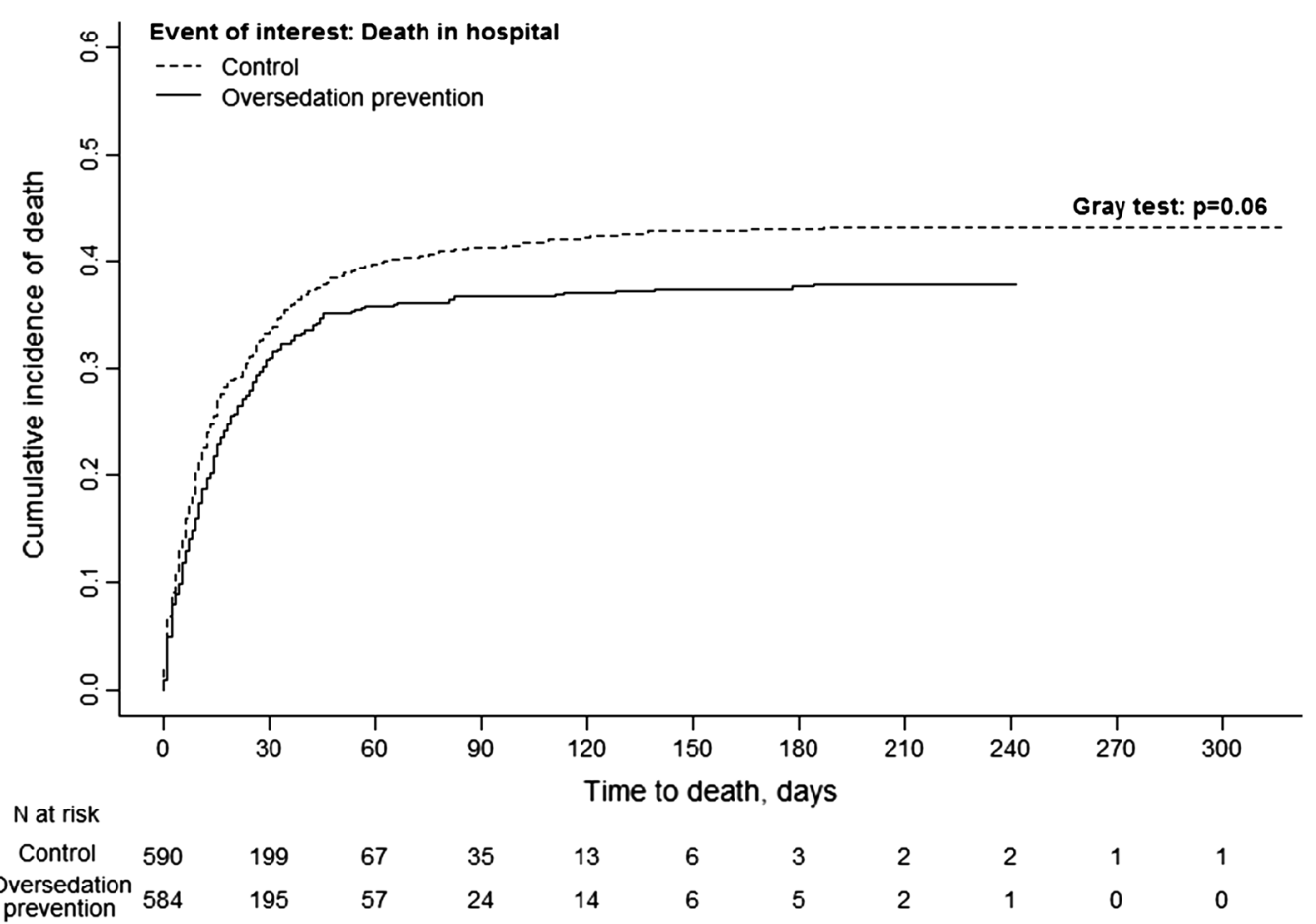

Fig. 3 Cumulative incidence of deaths in the hospital. The cumulative incidence of hospital death did not differ significantly between the two groups (220 in oversedation prevention group vs. 253 in the control group): hazard ratio for the oversedation prevention group versus the control group, $0.85 ; 95 \%$ confidence interval, $0.71-1.01 ; p=0.06$. For the analysis of time from randomization to death in the hospital, alive hospital discharge was handled as a competing risk

of consciousness base on a yes/no single-item scale, MV duration, etc.) all have their own limitations.

The oversedation prevention resulted in significantly less use of intravenous midazolam and propofol. Measuring doses of drug and showing a reduction in doses administered is not the same thing, but the increased rate of awake patients in the OSP group may imperfectly reflect the goal. Unfortunately, an explicit recording of implementation of multiple components of the OSP protocol could not be carried out in this large trial.

Of note, the lack of details regarding the previous alcohol consumption and the grouping within the large psychotropic category of medications with various mechanisms of action and side effects (benzodiazepines, neuroleptics, antidepressants, etc.) albeit pragmatic also represents a potential methodological limitation.

In summary, in this prospective randomized trial in severe critically ill patients requiring mechanical ventilation with early termination and under powering, we were unable to show that oversedation prevention significantly reduces mortality. However, it resulted in a significantly lower use of intravenous hypnotics, earlier time to spontaneous breathing trial, and reduced duration of mechanical ventilation. These last results should be interpreted with precaution regarding the several limitations of the trial including the early termination.

\section{Additional file}

Additional file 1. Complementary outcome criteria, results and SRLF trial group contributors.

\section{Abbreviations}

95\% Cl: 95\% confidence interval; HR: hazard ratio; ICU: intensive care unit; OSP: oversedation prevention.

\section{Authors' contributions}

Among the writing Committee members: Bernard DE JONGHE, Julie LEGER and Bruno GIRAUDEAU wrote the manuscript. Nadia AISSAOUI and Stephan EHRMANN participated in the critical revision of the article. The writing committee members, the CERC members, the investigators, the SRLF presidents, and secretaries (Additional file 1: Appendix 3 ) approved the final version to be published. All authors read and approved the final manuscript.

\section{Author details}

${ }^{1}$ Société de Réanimation de Langue Française (SRLF), 48 Avenue Claude Vellefaux, 75010 Paris, France.

\section{Acknowledgements}

The authors are deeply indebted to the patients who agreed to participate and to all physicians who took care of them. Special thanks to all involved in the collection and analysis of the data: Louise-Marie Laisne, all CERC members, all investigators, SRLF presidents and secretaries (Additional file 1: Appendix 3). 
We also thank Chantal Sevens, Florence Neels, and Mathieu Loung for their assistance.

\section{Competing interests}

The authors declare that they have no competing interests.

\section{Availability of data and materials}

The data set supporting the conclusions of this article is available by contacting the corresponding author.

\section{Consent for publication}

Not applicable.

\section{Ethics approval and consent to participate}

The protocol was approved by the local institutional ethics committee, and the study was performed in accordance with the ethical standards laid down in the 1964 Declaration of Helsinki.

\section{Funding}

The study was funded by the French Intensive Care Society (Société de Réanimation de langue française: SRLF Trial Group).

\section{Publisher's Note}

Springer Nature remains neutral with regard to jurisdictional claims in published maps and institutional affiliations.

Received: 11 January 2018 Accepted: 18 July 2018

Published online: 21 September 2018

\section{References}

1. Patel SB, Kress JP. Sedation and analgesia in the mechanically ventilated patient. Am J Respir Crit Care Med. 2012;185:486-97.

2. Jackson DL, Proudfoot CW, Cann KF, Walsh TS. The incidence of suboptimal sedation in the ICU: a systematic review. Crit Care. 2009;13:R204.

3. Kress J, Pohlman A, O'Connor M, Hall J. Daily interruption of sedative infusions in critically ill patients undergoing mechanical ventilation. $\mathrm{N}$ Engl J Med. 2000;342:1471-7.

4. Brook AD, Ahrens TS, Schaiff R, Prentice D, Sherman G, Shannon W, Kollef $\mathrm{MH}$. Effect of a nursing-implemented sedation protocol on the duration of mechanical ventilation. Crit Care Med. 1999;27:2609-15.

5. De Jonghe B, Bastuji-Garin S, Fangio P, Lacherade JC, Jabot J, Appere-DeVecchi C, Rocha N, Outin H. Sedation algorithm in critically ill patients without acute brain injury. Crit Care Med. 2005;33:120-7.

6. Quenot JP, Ladoire S, Devoucoux F, Doise JM, Cailliod R, Cunin N, Aube H, Blettery B, Charles PE. Effect of a nurse-implemented sedation protocol on the incidence of ventilator-associated pneumonia. Crit Care Med. 2007;35:2031-6.

7. Shehabi Y, Bellomo R, Reade MC, Bailey M, Bass F, Howe B, McArthur C, Murray L, Seppelt IM, Webb S, Weisbrodt L. Early goal-directed sedation versus standard sedation in mechanically ventilated critically ill patients: a pilot study*. Crit Care Med. 2013;41:1983-91.

8. Strom T, Martinussen T, Toft P. A protocol of no sedation for critically ill patients receiving mechanical ventilation: a randomised trial. Lancet. 2010;375:475-80.

9. Breen D, Karabinis A, Malbrain M, Morais R, Albrecht S, Jarnvig IL, Parkinson P, Kirkham AJ. Decreased duration of mechanical ventilation when comparing analgesia-based sedation using remifentanil with standard hypnotic-based sedation for up to 10 days in intensive care unit patients: a randomised trial. Crit Care. 2005:9:R200-10.

10. Treggiari MM, Romand JA, Yanez ND, Deem SA, Goldberg J, Hudson L, Heidegger CP, Weiss NS. Randomized trial of light versus deep sedation on mental health after critical illness. Crit Care Med. 2009;37:2527-34.

11. Shehabi Y, Bellomo R, Reade MC, Bailey M, Bass F, Howe B, McArthur CJ, Seppelt IM, Webb SA, Weisbrodt L. Early intensive care sedation predicts long-term mortality in ventilated critically ill patients. Am J Respir Crit Care Med. 2012;186:724-31.
12. Balzer F, Weiss B, Kumpf O, Treskatsch S, Spies C, Wernecke KD, Krannich A, Kastrup M. Early deep sedation is associated with decreased in-hospital and two-year follow-up survival. Crit Care. 2015;19:197.

13. Tanaka LM, Azevedo LC, Park M, Schettino G, Nassar AP, Rea-Neto A, Tannous L, de Souza-Dantas VC, Torelly A, Lisboa T, Piras C, Carvalho FB, Maia Mde O, Giannini FP, Machado FR, Dal-Pizzol F, de Carvalho AG, dos Santos RB, Tierno PF, Soares M, Salluh Jl. Early sedation and clinical outcomes of mechanically ventilated patients: a prospective multicenter cohort study. Crit Care. 2014;18:R156.

14. The SRLF Trial Group. Sedation in French intensive care units: a survey of clinical practice. Ann Intensive Care. 2013;3:24.

15. Boles JM, Bion J, Connors A, et al. Weaning from mechanical ventilation. Eur Respir J. 2007;29:1933-56.

16. Eldridge S, Kerry S, Torgerson DJ. Bias in identifying and recruiting participants in cluster randomised trials: What can be done? BMJ. 2009;339:b4006.

17. Giraudeau B, Ravaud P. Preventing bias in cluster randomised trials. PLoS Med. 2009;6:e1000065.

18. Torgerson DJ. Contamination in trials: Is cluster randomisation the answer? BMJ. 2001:322:355-7.

19. Savovic J, Jones HE, Altman DG, Harris RJ, Juni P, Pildal J, Als-Nielsen B, Balk EM, Gluud C, Gluud LL, loannidis JP, Schulz KF, Beynon R, Welton NJ, Wood L, Moher D, Deeks JJ, Sterne JA. Influence of reported study design characteristics on intervention effect estimates from randomized, controlled trials. Ann Intern Med. 2012;157:429-38.

20. Fine JP, Gray RJ. A proportional hazards model for the subdistribution of a competing risk. J Am Stat Assoc. 1999:94:496-509.

21. R-Core-Team. R: a language and environment for statistical computing. 2015. https://www.R-project.org. Accessed 17 Sept 2018.

22. Wunsch H, Kahn JM, Kramer AA, Rubenfeld GD. Use of intravenous infusion sedation among mechanically ventilated patients in the United States. Crit Care Med. 2009;37:3031-9.

23. Pandharipande PP, Pun BT, Herr DL, Maze M, Girard TD, Miller RR, Shintani AK, Thompson JL, Jackson JC, Deppen SA, Stiles RA, Dittus RS, Bernard GR, Ely EW. Effect of sedation with dexmedetomidine vs lorazepam on acute brain dysfunction in mechanically ventilated patients: the MENDS randomized controlled trial. JAMA. 2007;298:2644-53.

24. Riker RR, Shehabi Y, Bokesch PM, Ceraso D, Wisemandle W, Koura F, Whitten P, Margolis BD, Byrne DW, Ely EW, Rocha MG. Dexmedetomidine vs midazolam for sedation of critically ill patients: a randomized trial. JAMA. 2009;301:489-99.

25. Jakob SM, Ruokonen E, Grounds RM, Sarapohja T, Garratt C, Pocock SJ Bratty JR, Takala J. Dexmedetomidine vs. midazolam or propofol for sedation during prolonged mechanical ventilation: two randomized controlled trials. JAMA. 2012;307:1151-60.

26. Chanques $G$, Jaber S, Barbotte E, Violet $S$, Sebbane M, Perrigault PF, Mann C, Lefrant JY, Eledjam JJ. Impact of systematic evaluation of pain and agitation in an intensive care unit. Crit Care Med. 2006;34:1691-9.

27. Boles JM, Bion J, Connors A, Herridge M, Marsh B, Melot C, Pearl R, Silverman $H$, Stanchina M, Vieillard-Baron A, Welte T. Weaning from mechanical ventilation. Eur Respir J. 2007;29:1033-56.

28. Girard TD, Kress JP, Fuchs BD, Thomason JW, Schweickert WD, Pun BT, Taichman DB, Dunn JG, Pohlman AS, Kinniry PA, Jackson JC, Canonico AE, Light RW, Shintani AK, Thompson JL, Gordon SM, Hall JB, Dittus RS, Bernard GR, Ely EW. Efficacy and safety of a paired sedation and ventilator weaning protocol for mechanically ventilated patients in intensive care (Awakening and Breathing Controlled trial): a randomised controlled trial. Lancet. 2008;371:126-34.

29. Elliott R, McKinley S, Aitken LM, Hendrikz J. The effect of an algorithmbased sedation guideline on the duration of mechanical ventilation in an Australian intensive care unit. Intensive Care Med. 2006:32:1506-14.

30. Eldridge S, Kerry S, Torgerson DJ. Bias in identifying and recruiting participants in cluster randomised trials: What can be done? BMJ. 2009;339:b4006.

31. Giraudeau B, Ravaud P. Preventing bias in cluster randomised trials. PLoS Med. 2009:6:e1000065.

32. Torgerson DJ. Contamination in trials: Is cluster randomisation the answer? BMJ. 2001;322:355-7. 